\title{
Probing millicharged particles with NA64 experiment at CERN
}

\author{
S. N. Gninenko, ${ }^{1, *}$ D. V. Kirpichnikov, ${ }^{1, \dagger}$ and N. V. Krasnikov ${ }^{1,2, \$}$ \\ ${ }^{1}$ Institute for Nuclear Research of the Russian Academy of Sciences, 117312 Moscow, Russia \\ ${ }^{2}$ Joint Institute for Nuclear Research, 141980 Dubna, Russia
}

(Received 20 March 2019; published 6 August 2019)

\begin{abstract}
In this paper we estimate the sensitivity of the NA64 experiment to millicharged particles $(\chi)$. That experimental facility is dedicated to the searching for dark sector particles in missing energy events at the CERN SPS. We consider missing momentum signatures in the $\simeq 100 \mathrm{GeV}$ electron and muon beams and show that the later one allows to obtain more stringent bounds on the millicharge $Q_{\chi}$, which for the $\chi$ masses $100 \mathrm{MeV} \leq m_{\chi} \leq 500 \mathrm{MeV}$ at the level $Q_{\chi} / e \lesssim O\left(10^{-3}\right)-O\left(10^{-2}\right)$.
\end{abstract}

DOI: $10.1103 /$ PhysRevD.100.035003

\section{INTRODUCTION}

The millicharged particles $(\chi)$, i.e., particles with an electric charge $Q_{\chi}=\epsilon e$ much smaller $(\epsilon \ll 1)$ than the elementary charge $e$, have been considered long ago. Those particles were discussed in connection with the mechanism of the electric charge quantization and a possible nonconservation of the electric charge [1]. In the context of grand unification models this mechanism may be linked to magnetic monopole and electric charge quantization [2]. However, the magnetic monopoles have not been observed yet, and the underlying mechanism for charge quantization remains nonconfirmed, thus making searches for millicharged particles of great interest.

The $\chi$ s have been also proposed in various extensions of the Standard Model (SM). In particular, in the hidden (dark) sector models with a new $U^{\prime}(1)$ gauge group [3], see also [4]. In this scenario the kinetic mixing between the $U^{\prime}(1)$ and the SM fields is described by a term $\frac{\epsilon}{2} F_{\mu \nu}{ }^{\prime} F^{\mu \nu}$, where $F_{\mu \nu}^{\prime}=\partial_{\mu} A_{\nu}^{\prime}-\partial_{\nu} A_{\nu}^{\prime}$ and $F_{\mu \nu}=\partial_{\mu} A_{\nu}-\partial_{\nu} A_{\nu}$ with $A_{\mu}^{\prime}$ and $A_{\mu}$ being a dark photon and ordinary photon respectively. After a redefinition of the hidden vector field $A_{\mu}^{\prime} \rightarrow A_{\mu}^{\prime}+\epsilon A_{\mu}$ one can find that electromagnetic field $A_{\mu}$ interacts with hidden fermions of the dark sector, namely $\mathcal{L}_{\text {int }}=\epsilon A_{\mu} J_{D}^{\mu}$. Here $J_{D}^{\mu}=g_{D} \bar{\chi} \gamma^{\mu} \chi$ is the $U^{\prime}(1)$ current of the fermions from the dark sector. This means that the dark sector particles $\chi$ interact with the photon via the effective coupling $Q_{\chi}=\epsilon g_{D}$.

\footnotetext{
*sergei.gninenko@cern.ch

${ }^{\dagger}$ kirpich@ms2.inr.ac.ru

‡nikolai.krasnikov@cern.ch
}

Published by the American Physical Society under the terms of the Creative Commons Attribution 4.0 International license. Further distribution of this work must maintain attribution to the author(s) and the published article's title, journal citation, and DOI. Funded by SCOAP.
In this scenario the dark photon remains massless and interacts only with dark sector particles. In the rest of this work, we will consider the following Lagrangian for the $\chi \mathrm{s}$ interacting with the electromagnetic field $A_{\mu}$, assuming that they are spin $1 / 2$ fermions:

$$
\mathcal{L} \supset i \bar{\chi} \gamma^{\mu} \partial_{\mu} \chi-m_{\chi} \bar{\chi} \chi+Q_{\chi} A_{\mu} \bar{\chi} \gamma^{\mu} \chi
$$

where $m_{\chi}$ is a Dirac mass of the hidden particles.

As it follows from (1) in the leading order the $\chi$ production rate is proportional to $Q_{\chi}^{2}$ and the $\chi$ s can be effectively produced in any electromagnetic reactions if kinematically allowed [5]. The numerous constraints on $Q_{\chi}$ are obtained from the dedicated beam-dump [6,7], positronium [8] and reactor $[9,10]$ experiments, see Ref. [11] for a review. The expected limits from the $e^{+} e^{-}$colliders and the LHC $[12,13]$ have been reported recently. Stringent constraints on $\chi$ can also be obtained from cosmological and astrophysical considerations, see e.g., [11,14-20].

The millicharged particles with $Q_{\chi} \ll e$ typically escape the detection in an experiment, ${ }^{1}$ because their ionization energy loss is $\sim Q_{\chi}^{2}$ and thus is very small. Therefore, to observe them directly a large number of particles on target is required, see e.g., [7,21]. However, possible indirect observation of $\chi \mathrm{s}$ at the fixed-target facilities can utilize another more effective approach-the search for the $\chi \mathrm{s}$ in missing energy/momentum events [22-25].

Let us consider the NA64 experiment at CERN [23,2628], which was designed to search for the light dark matter particles in the reaction of dark photon production $e Z \rightarrow$ $e Z A^{\prime}$ followed by the invisible decay of dark photon into hidden states, $A^{\prime} \rightarrow$ invisible. However, the missing energy signature for the search of dark photons can also be

\footnotetext{
${ }^{1}$ Here $e$ is the electron electric charge, $\frac{e^{2}}{4 \pi}=\frac{1}{137}$.
} 
implemented to search for the millicharged particles produced in the similar reaction $e Z \rightarrow e Z \chi \bar{\chi}$. At present the NA64 experiment uses the electron beam with the energy $E_{0} \approx 100 \mathrm{GeV}$, but there are also plans to use the high intensity muon M2 beam line at the Super Proton Synchrotron (SPS) at CERN [29]. Moreover, the missing momentum experiments with muon beams at CERN [30-32] and FermiLab $[33,34]$ have been proposed recently in order to probe the $(g-2)_{\mu}$ anomaly [35] in the framework of the light dark matter sector $[24,25]$.

It should be noted that the LHC experiments are insensitive to probe the sub-GeV dark sector scenario [36] with small coupling constants. In particular, the millicharge parameter space in the ranges $0.1 \mathrm{GeV} \lesssim m_{\chi} \lesssim$ $1 \mathrm{GeV}$ and $10^{-4} \lesssim Q_{\chi} / e \lesssim 10^{-3}$ has not been constrained yet by existing experiments. The scenarios of sub-GeV hidden particles can be probed at the SHIP [37] proton beam dump facility as well as at MiniBoone [38], DUNE [39] and LSND [40] neutrino detectors. In these experiments the dominant millicharge production signatures are exotic decays:

$$
\pi^{0} / \eta \rightarrow \gamma \chi \bar{\chi}, \quad J / \psi, \Upsilon \rightarrow \chi \bar{\chi} .
$$

The produced millicharged particles elastically scatter on atomic electrons in the dump, $\chi e \rightarrow \chi e$. So the detection of millicharged particles is based on the measurement of low energy electron recoils. The millicharge yield from hadrons [21] is suppressed by both the production term $\sim Q_{\chi}^{2}$ and the interaction factor $\sim Q_{\chi}^{2}$, such that $N_{\chi \chi} \sim Q_{\chi}^{4}$. On the other hand, the number of produced millicharged particles at NA64 is proportional to $Q_{\chi}^{2}$ for both electron and muon beams. For the muon beam significant gains in millicharge sensitivity compared to electron beam may be achieved by optimizing the active target design of NA64. In particular, $10^{13}$ muons on target are expected to accumulate at NA64 during the couple of months running.

In this note we estimate the discovery potential of millicharged particles at the NA64 experiment for both electron and muon beams. We find that muon beam setup of NA64 provides more stringent bounds on electric charge $Q_{\chi}$ in comparison with electron beam. The main reason is that $100-\mathrm{GeV}$ electron beam degrades significantly even in the relatively thin lead target of $40 X_{0}(\approx 20 \mathrm{~cm})$ used at NA64. ${ }^{2}$ Therefore, the electron missing momentum yield is suppressed by the electron beam attenuation factor $X_{0}$ and the number of produced millicharged particles is proportional to $N_{\chi \chi} \sim X_{0}$. On the other hand muon radiation length is $X_{0}^{\mu} \sim\left(m_{\mu} / m_{e}\right)^{2} X_{0} \gg X_{0}$, thus the relativistic $100 \mathrm{GeV}$ muons pass through the dump with $L \ll X_{0}^{\mu}$ without significant loss of muon energy. This implies that the

\footnotetext{
${ }^{2}$ Here $X_{0}$ is electron radiation length.
}

millicharge production signal in the muon beam experiment is proportional to the length of the target, $N_{\chi \chi} \sim 40 X_{0}{ }^{3}$ Furthermore, one can improve the millicharge sensitivity for the muon beam by increasing the effective interacting length of the active lead target. For instance, by increasing the length of the target by a factor of 4 magnitude, one can extend the $Q_{\chi}$ bound by a factor of 2 . This provides an excellent opportunity for NA64 with muon beam to probe a wider range of millicharge parameter space. We also derive the NA64 bound on millicharges from the recent NA64 experimental bound on the $\epsilon$ parameter [27] for the dark photon model.

The organization of the paper is as follows. In Sec. II we collect basic formulas which are relevant for an estimation of the millicharged particles production rates. In Sec. III we present the bound on $\left(Q_{\chi}, m_{\chi}\right)$ from the NA64e experiment. In Sec. IV we discuss the expected limits from combined analysis of NA64e and NA64 $\mu$. The last section summarizes the main results.

\section{THE CROSS SECTIONS}

In this section we present basic formulas for the cross section of the high-energy lepton scattering on heavy nuclei accompanied by the emission of a bremsstrahlung $\chi \bar{\chi}$ pair,

$$
l N \rightarrow l N \gamma^{*} \rightarrow l N \chi \bar{\chi}
$$

with $\chi$ being a millicharged Dirac fermion and $l=e, \mu$. The relevant tree level diagrams are shown in Fig. 1 for the muon case. The dominant millicharge emission cross section (3) is proportional to $\mathcal{O}\left(\alpha^{3} Q_{\chi}^{2}\right)$. We neglect the $\mathcal{O}\left(\alpha^{2} Q_{\chi}^{4}\right)$ trident millicharge production cross section and relevant $\mathcal{O}\left(\alpha^{5 / 2} Q_{\chi}^{3}\right)$ interference terms in our calculations. Therefore, the differential cross section $\sigma(l N \rightarrow l N \chi \bar{\chi})$ can be represented in the form [41]

$d \sigma(l N \rightarrow l N \chi \bar{\chi})=d \operatorname{Lips}_{2 \rightarrow 3} \overline{\left|\mathcal{M}_{2 \rightarrow 3}\right|^{2}}{ }_{\alpha \beta} \frac{d k_{\gamma^{*}}^{2}}{(2 \pi)} \times \chi_{\alpha \beta}$,

where $d \operatorname{Lips}_{2 \rightarrow 3}$ is the Lorentz invariant phase space for a process $l N \rightarrow l N \gamma^{*}$ with the off-shell photon in the final state,

$$
\overline{\left|\mathcal{M}_{2 \rightarrow 3}\right|^{2}}{ }_{\alpha \beta}=\overline{\sum_{\text {ppin }}} \mathcal{M}^{\mu} \mathcal{M}^{\nu \dagger} g_{\mu \alpha} g_{\nu \beta} \cdot \frac{1}{k_{\gamma^{*}}^{4}},
$$

where an averaging over initial lepton spin and summation over the outgoing lepton state is performed. The millicharged tensor $\chi_{\alpha \beta}$ has the following form:

\footnotetext{
${ }^{3}$ Here as an estimate we use the length of the target for the muon experiment $L \sim 40 X_{0} \approx 20 \mathrm{~cm}$.
} 


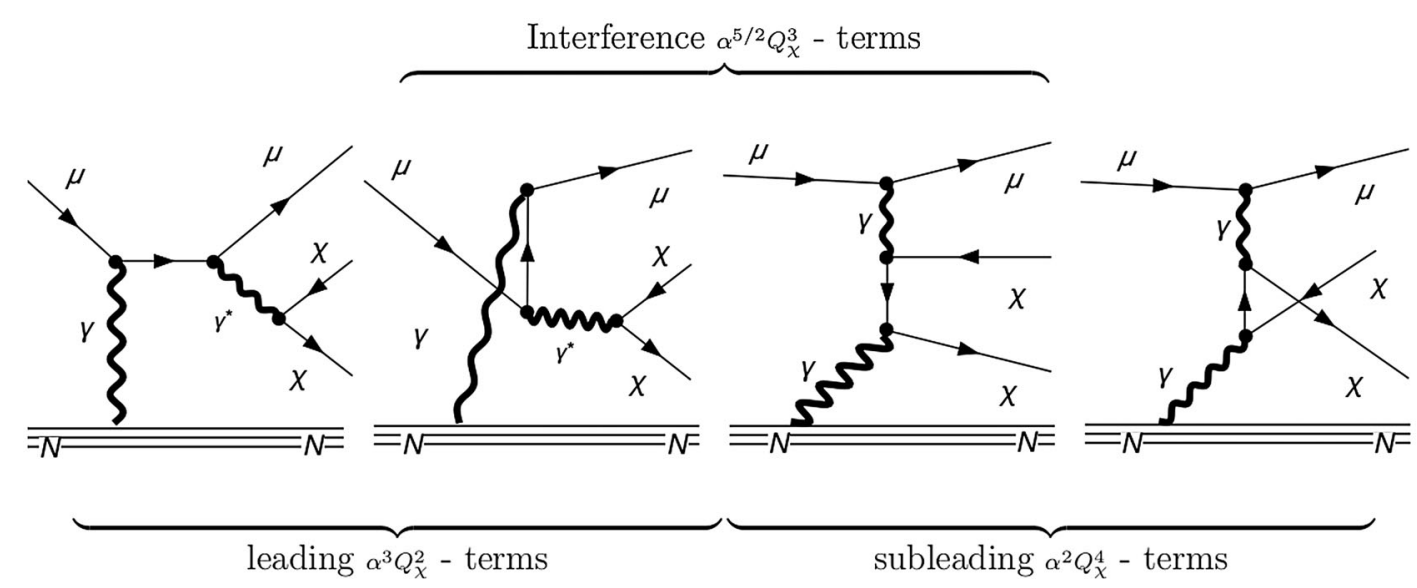

FIG. 1. Feynman diagrams of millicharge pair production.

$$
\begin{aligned}
\chi_{\alpha \beta}= & \int \frac{d^{3} \mathbf{k}_{1}}{(2 \pi)^{3} 2 E_{1}} \frac{d^{3} \mathbf{k}_{2}}{(2 \pi)^{3} 2 E_{2}}(2 \pi)^{4} \delta^{(4)}\left(k-k_{1}-k_{2}\right) \\
& \times \sum j_{\alpha} j_{\beta}^{*},
\end{aligned}
$$

with $j_{\alpha}=Q_{\chi} \bar{\chi} \gamma_{\alpha} \chi$ being a millicharged current, $k \equiv k_{\gamma^{*}}$ is a total four-momentum of the millicharged pair, $k_{\gamma^{*}}=$ $k_{1}+k_{2}$. This implies that the millicharge production cross section $d \sigma(l N \rightarrow l N \chi \bar{\chi})$ can be represented in the form

$$
\begin{aligned}
d \sigma(l N \rightarrow l N \chi \bar{\chi})= & d \sigma\left(l N \rightarrow l N \gamma^{*}\right) \\
& \times \frac{Q_{\chi}^{2}}{12 \pi^{2}} \frac{d k_{\gamma^{*}}^{2}}{k_{\gamma^{*}}^{2}} \sqrt{1-\frac{4 m_{\chi}^{2}}{k_{\gamma^{*}}^{2}}}\left(1+\frac{2 m_{\chi^{2}}}{k_{\gamma^{*}}^{2}}\right) .
\end{aligned}
$$

The first factor in (7) can be calculated in the equivalent photon approach $[42,43]$; the corresponding differential cross section is

$$
\begin{aligned}
\frac{d}{d x} \sigma_{2 \rightarrow 3} \approx & \frac{2}{3} \frac{\alpha^{3} \zeta\left|\mathbf{k}_{\gamma^{*}}\right|}{x \tilde{u}^{2} E_{0}}\left[m_{l}^{2} x\left(-2+2 x+x^{2}\right)\right. \\
& \left.-2\left(3-3 x+x^{2}\right) \tilde{u}\right], \quad x=E_{\gamma^{*}} / E_{0}
\end{aligned}
$$

with $\zeta$ being the photon flux from nucleus

$$
\zeta=\int_{k_{\gamma^{*}}^{4} /\left(4 E_{0}^{2}\right)}^{k_{\gamma^{*}}^{2}+m_{l}^{2}} \frac{d t}{t^{2}}\left[t-\frac{k_{\gamma^{*}}^{2}}{4 E_{0}^{2}}\right] \cdot Z^{2}\left(\frac{a^{2} t}{a^{2} t+1}\right)^{2} \frac{1}{(1+t / d)^{2}},
$$

where $a=111 Z^{-1 / 3} / m_{e}$ parametrizes the electron screening effect and $d=0.164 A^{-2 / 3} \mathrm{GeV}^{2}$ stands to account the finite nuclear size. Such form-factor parametrization (9) accounts for elastic scattering effects only. The inelastic form factor is proportional to $\sim Z$ and thus can be neglected in the high- $Z$ target experiment. The quantities $\tilde{u}$ and $\left|\mathbf{k}_{\gamma^{*}}\right|$ in (8) are defined by $\tilde{u}=-k_{\gamma^{*}}^{2}(1-x) / x-m_{l}^{2} x$ and $\left|\mathbf{k}_{\gamma^{*}}\right|=$ $\left(x^{2} E_{0}^{2}-k_{\gamma^{*}}^{2}\right)^{1 / 2}$ respectively. Therefore, one can estimate the $\chi \bar{\chi}$-production rate by integrating (7) over $\gamma^{*}$ invariant mass,

$$
\begin{aligned}
\sigma_{l N \rightarrow l N \chi \bar{\chi}} & \approx \int_{0.5}^{1} d x \int_{1}^{y_{\max }} \frac{d y}{y} \sqrt{1-\frac{1}{y}}\left(1+\frac{1}{2 y}\right) \\
& \times \frac{Q_{\chi}^{2}}{12 \pi^{2}} \times \frac{d \sigma_{2 \rightarrow 3}}{d x},
\end{aligned}
$$

where we denote $y=k_{\gamma^{*}}^{2} /\left(4 m_{\chi}^{2}\right)$ and $y_{\max }=x^{2} E_{0}^{2} /\left(4 m_{\chi}^{2}\right)$. Here the lower limit in the integration over $x$ corresponds to the following missing energy cut, $E_{\text {miss }} / E_{0} \equiv E_{\gamma^{*}} /$ $E_{0}>1 / 2$. It is instructive to obtain the approximate expression for (10). Indeed, the integral over $x$ in (10) can be estimated in a way analogous to that performed in [43]. Namely, for $k_{\gamma^{*}}>m_{l}$ the integral saturates at $x \approx 1$, therefore one has

$$
\int_{0.5}^{1} d x \frac{d \sigma_{2 \rightarrow 3}}{d x} \approx \frac{4}{3} \frac{\alpha^{3} \zeta}{\left(k_{\gamma^{*}}\right)^{2}}\left[\ln \frac{1}{2}\left(\frac{k_{\gamma^{*}}}{m_{l}}\right)^{2}+\mathcal{O}(1)\right],
$$

where $k_{\gamma^{*}} / m_{l}$ under the logarithm regulates a soft lepton singularity. The integration over $y$ can be performed in the leading logarithmic order. So for $m_{\chi} \gtrsim m_{l}$ the cross section (10) can be approximated with a logarithmic accuracy as

$\sigma(l N \rightarrow l N \chi \bar{\chi}) \approx \frac{4}{3} \frac{\alpha^{3} \zeta}{\left(2 m_{\chi}\right)^{2}}\left[\ln \frac{1}{2}\left(\frac{2 m_{\chi}}{m_{l}}\right)^{2}+\mathcal{O}(1)\right] \kappa \frac{Q_{\chi}^{2}}{12 \pi^{2}}$,

where $\kappa$ is a function which depends weakly on $m_{\chi} / E_{0}$. Namely, for $m_{\chi} / E_{0} \ll 1$, one has $y_{\max } \gg 1$, so that the integral for $\kappa$ can be calculated straightforwardly: 


$$
\kappa \approx \int_{1}^{\infty} \frac{d y}{y^{2}} \sqrt{1-\frac{1}{y}}\left(1+\frac{1}{2 y}\right)=\frac{4}{5} .
$$

In addition, we note that (12) generally resembles, up to the numerical factor $\sim Q_{\chi}^{2}$ and additive correction to the logarithm, the total cross section for the dark photon production [43], in which the dark photon mass is redefined as $m_{A^{\prime}} \rightarrow 2 m_{\chi}$. This observation allows one to estimate analytically the expected constraints for the parameter space of the millicharged particles directly for muon and electron beam at NA64.

\section{LIMITS FROM NA64e}

One can link the bound on the $\epsilon$ parameter for the model with dark photon and bound on the millicharge. Indeed, the interaction of the dark photon with the SM particles has the form [43]

$$
\mathcal{L}_{\text {dark }}=\epsilon e J_{\mathrm{SM}}^{\mu} A_{\mu}^{\prime},
$$

where $J_{\mathrm{SM}}^{\mu}=\frac{2}{3} \bar{u} \gamma^{\mu} u-\frac{1}{3} \bar{d} \gamma^{\mu} d-\bar{e} \gamma^{\mu} e+\cdots$ is the SM electromagnetic current, $\frac{e^{2}}{4 \pi}=\frac{1}{137}, A_{\mu}^{\prime}$ is the dark photon field and $\epsilon$ is the unknown parameter. The goal of the experiments is to derive the bound on $\epsilon$. On the other hand, the bound on $\epsilon$ depends on the dark photon mass $m_{A^{\prime}}$. The cross section of dark photon electroproduction $\sigma\left(e N \rightarrow e N A^{\prime}\right)$ is proportional to the cross section of virtual photon electroproduction, namely [43]

$$
\begin{aligned}
\sigma\left(e N \rightarrow e N A^{\prime}\right) & =\epsilon^{2} \sigma\left(e N \rightarrow e N \gamma^{*}\right) \\
& \approx \frac{4}{3} \frac{\alpha^{3} \epsilon^{2} \zeta}{m_{A^{\prime}}^{2}}\left[\ln \frac{1}{2}\left(\frac{m_{A^{\prime}}}{m_{e}}\right)^{2}+\mathcal{O}(1)\right],
\end{aligned}
$$

where $m_{A^{\prime}}^{2}=k_{\gamma^{*}}^{2}$ is the four momentum squared of virtual photon.

In a recent NA64e analysis [27] stringent experimental constraints on the dark photon coupling, $10^{-5}<\epsilon<10^{-2}$, for the mass range $m_{A^{\prime}} \lesssim 1 \mathrm{GeV}$ were derived. These bounds were obtained by using the GEANT4 Monte Carlo simulation for the flux and spectra of the $A^{\prime}$ s produced in the target by primary electrons. That numerical simulation takes into account the development of the signal electromagnetic (EM) shower in the reaction $e N \rightarrow e N A^{\prime} \rightarrow e N \chi \chi$ in the target. We emphasize that the dark photons and millicharged pairs are essentially produced within the first radiation length of the target material for the electron beam. Therefore, the beam attenuation and EM shower development should be taken into account in proper numerical simulations of $\chi$-pair production. The latter is beyond of the scope of our paper. We leave this task for future analysis. Instead, in order to obtain the 95\% C.L. bound on $Q_{\chi}^{(e)}$ we link it with an upper bound $\epsilon^{2}$ from Ref. [27] as

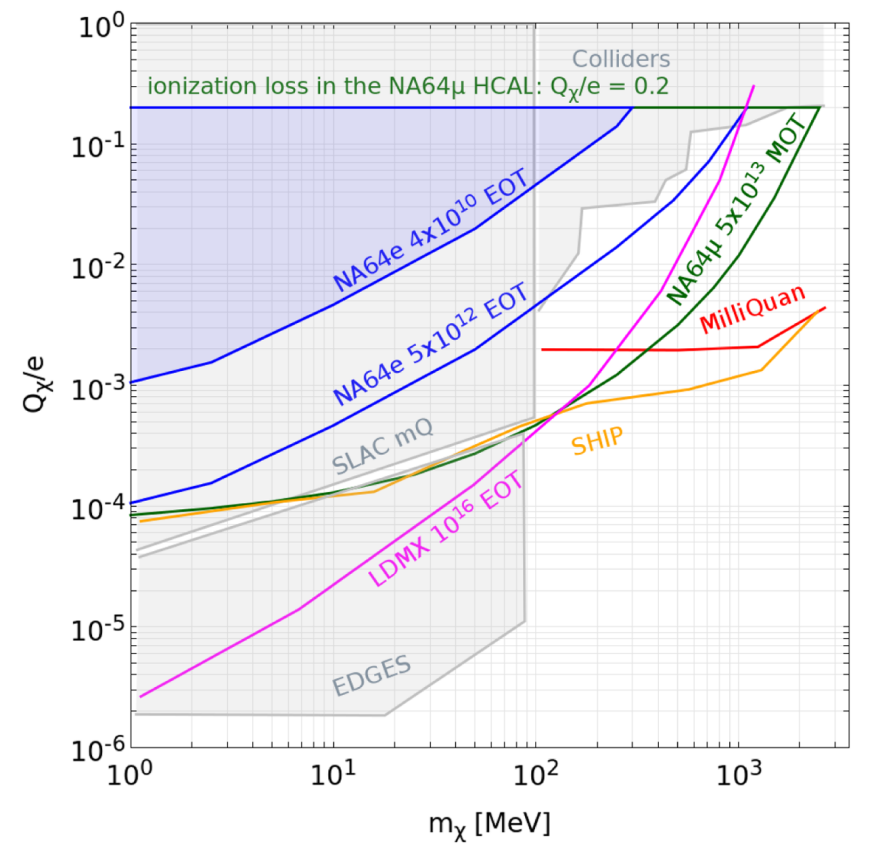

FIG. 2. Upper limits on the fractional electric charge $Q_{\chi} / e$ of the hypothetical millicharged fermions of mass $m_{\chi}$. The areas with the grey shading are the bounds excluded by SLAC [7], collider [44,45] and EDGES experiment [46,47]. The projected limits are shown by solid lines. In particular, the expected reaches for SHIP and MilliQuan are taken from [21]. The sensitivity of LDMX is based on the MADGRAPH missing momentum simulation with $16 \mathrm{GeV}$ electron beam on the aluminum target [24,25]. The blue shaded region is the bound experimentally excluded by NA64, see e.g., Ref. [27]. The upper bound at $Q_{\chi} / e=0.2$ corresponds to the (90\% C.L.) lower limit on the charge of $\chi$ 's above which they are detected in the Hadron calorimeter of NA64.

$$
\epsilon^{2} \approx \kappa \cdot \frac{\left(Q_{\chi}^{(e)}\right)^{2}}{12 \pi^{2}}
$$

which also implies the replacement $m_{A^{\prime}} \rightarrow 2 m_{\chi}$ in the relevant exclusion plot. Indeed, one can easily obtain (16) from Eqs. (12) and (15). We expect that the $\epsilon^{2}$ limit in (16) takes into account the processes associated with EM shower development in the thick target for the same kinematical cuts of energy deposition. In particular, in the present analysis the missing energy cut [27] for the dark photon search, $E_{A^{\prime}} \geq 0.5 E_{0}$, is associated with the cut for the millicharged particles search, $E_{\chi}+E_{\bar{\chi}} \geq 0.5 E_{0}$. In Fig. 2 we show the relevant excluded area by the blue shaded region.

\section{COMBINED EXPECTED BOUNDS FROM NA64e AND NA64 $\mu$}

In this section we estimate expected bounds on the charge of millicharged particles using the results of Secs. II and III. For the thin target with $L_{T} \ll X_{0}^{\mu}$ the millicharge yield, which originates from the $\mu Z \rightarrow \mu Z \chi \chi$ process, can be estimated as 


$$
N_{\chi \chi}=N_{\text {MOT }} \times \frac{\rho \times N_{A}}{A} \times L_{T} \times \sigma_{\chi \chi}
$$

where $N_{\text {MOT }}$ is a number of muons on target, $A$ is the atomic weight, $N_{A}$ is Avogadro's number, $\rho$ denotes the target density and $\sigma_{\chi \chi}$ is the millicharged pair production cross section (10). In our estimates we assume that the muon beam energy is about $100 \mathrm{GeV}$ and the muon flux is about $N_{\text {MOT }}=5 \times 10^{13}$. We consider lead target with thickness of $L_{T}=40 X_{0}=20 \mathrm{~cm}$. We neglect muon energy losses in the lead target. Indeed, this approximation is reasonable, because the muon energy attenuation reported in [34] is rather small for the beam energy range, $\left\langle d E_{\mu} / d z\right\rangle \approx 12.7 \times 10^{-3} \mathrm{GeV} / \mathrm{cm}$. We assume that the energies of initial and final muons are known. In the NA64 facility with muon beam it is assumed to utilize two, upstream and downstream, magnetic spectrometers allowing for precise measurements of momenta for incident and recoiled muons, respectively [30]. The missing energy signature can be used for the search for pair produced millicharged particles in the millicharged production reaction $\mu N \rightarrow \mu N \chi \chi$ in a way analogous to the case of dark photon searching.

Using the formula (17) for the number of produced millicharged particles and the expression (10) for the production cross section, we find the expected bound on millicharge $Q_{\chi}{ }^{4}$ We require $N_{\chi \chi}>2.3$ that corresponds to the $90 \%$ C.L. exclusion limit on $Q_{\chi} / e$. In Fig. 2 we show the expected reach of the NA64 detector for $N_{\text {MOT }}=5 \times 10^{13}$ muons and $N_{\mathrm{EOT}}=5 \times 10^{12}$ electrons respectively (here $N_{\text {EOT }}$ is a number of electrons on target), we assume that the beam energy is $E_{0}=100 \mathrm{GeV}$ for both $e$ and $\mu$ modes.

It is instructive to compare qualitatively millicharge limits for muon and electron beam in order to understand why the expected bound from muon setup is enhanced at $m_{\chi} \gtrsim m_{\mu}$. Indeed, the ratio of the reaches can be naively approximated as follows:

$$
\frac{Q_{\chi}^{(e)}}{Q_{\chi}^{(\mu)}} \approx\left(\frac{L_{\mathrm{eff}}^{(\mu)}}{L_{\mathrm{eff}}^{(e)}} \cdot \frac{N_{\mathrm{MOT}}}{N_{\mathrm{EOT}}} \cdot \frac{\sigma_{\chi \chi}^{(\mu)}\left[Q_{\chi}^{(\mu)}=1\right]}{\sigma_{\chi \chi}^{(e)}\left[Q_{\chi}^{(e)}=1\right]}\right)^{1 / 2},
$$

where labels $(\mu / e)$ specify a beam type and $L_{\text {eff }}$ is the effective length of millicharge leptoproduction in the lead target, namely $L_{\text {eff }}^{(\mu)} \approx 40 X_{0}$ and $L_{\text {eff }}^{(e)} \approx X_{0} \approx 0.5 \mathrm{~cm}$. The latter means that for electron beam the millicharges are essentially produced in the length $L \lesssim X_{0}$ of the target due to the large electron energy loss, while the muons produce millicharges uniformly over the whole length of the target. For beam energy $E_{0}=100 \mathrm{GeV}$ and millicharge masses $m_{\chi} \gtrsim 200 \mathrm{MeV}$ the electron and muon cross sections scale respectively as [43]

\footnotetext{
${ }^{4}$ We assume background free regime, that looks reasonable, see [30].
}

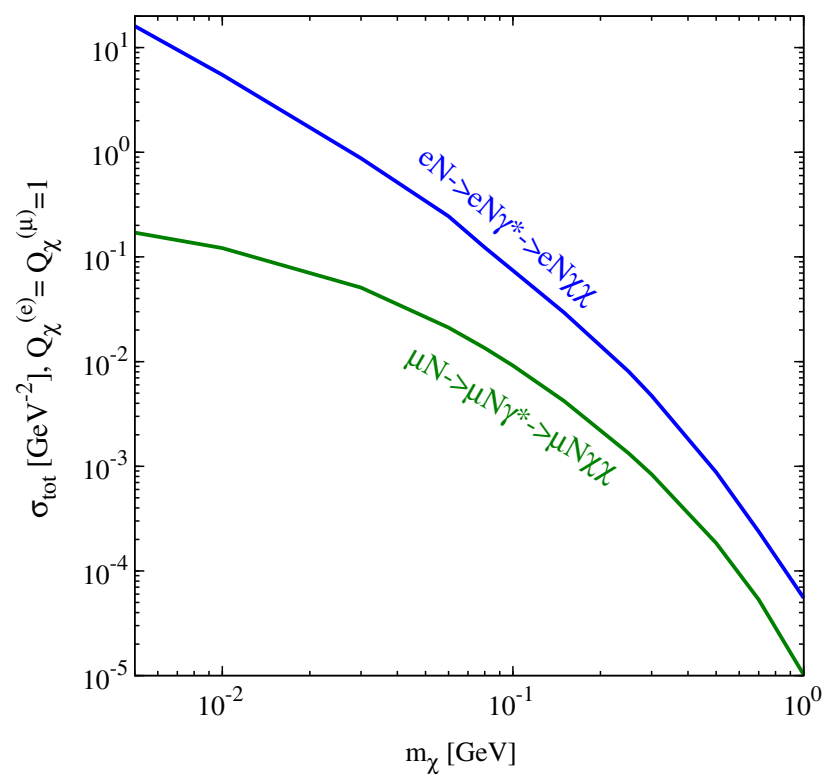

FIG. 3. The total production cross sections of millicharged particles as a function of their mass for the lead target and initial beam energy of $100 \mathrm{GeV}$. The green and blue curves show the cross sections for muon and electron beam respectively. The cross sections are shown for $Q_{\chi}^{(\mu)}=Q_{\chi}^{(e)}=1$.

$$
\begin{aligned}
\sigma_{\chi \chi}^{(e)} & \sim \frac{Q_{\chi}^{2}}{\left(2 m_{\chi}\right)^{2}}\left(\ln \frac{1}{2}\left[\frac{E_{0}}{2 m_{\chi}}\right]^{2}+\mathcal{O}(1)\right), \\
\sigma_{\chi \chi}^{(\mu)} & \sim \frac{Q_{\chi}^{2}}{\left(2 m_{\chi}\right)^{2}}\left(\ln \frac{1}{2}\left[\frac{2 m_{\chi}}{m_{\mu}}\right]^{2}+\mathcal{O}(1)\right),
\end{aligned}
$$

where factor $1 / 2$ under the logarithms comes from the integration of production cross section over the missing energy range, $1 / 2<E_{\gamma^{*}} / E_{0}<1$. For $N_{\mathrm{MOT}}=5 \times 10^{13}$ and $N_{\mathrm{EOT}}=5 \times 10^{12}$ one has $Q_{\chi}^{(e)} / Q_{\chi}^{(\mu)} \approx 9$ at $m_{\chi}=$ $200 \mathrm{MeV}$. The corresponding result is shown in Fig. 2. Indeed, for $m_{\chi}=200 \mathrm{MeV}$ we have $Q_{\chi}^{(e)} / e \approx 10^{-2}$ and $Q_{\chi}^{(\mu)} / e \approx 10^{-3}$. One can also estimate the ratio (18) for relatively light millicharges $m_{\chi} \ll m_{\mu}$. In this case muons produce the millicharges in the bremsstrahlung-like limit. The cross sections for both electron and muon beam can be approximated as

$$
\begin{aligned}
\sigma_{\chi \chi}^{(e)} & \sim \frac{Q_{\chi}^{2}}{\left(2 m_{\chi}\right)^{2}}\left(\ln \frac{1}{2}\left[\frac{2 m_{\chi}}{m_{e}}\right]^{2}+\mathcal{O}(1)\right), \\
\sigma_{\chi \chi}^{(\mu)} & \sim \frac{Q_{\chi}^{2}}{m_{\mu}^{2}}\left(\ln \frac{1}{2}\left[\frac{m_{\mu}}{2 m_{\chi}}\right]^{2}+\mathcal{O}(1)\right) .
\end{aligned}
$$

As a result we get $Q_{\chi}^{(e)} \gtrsim Q_{\chi}^{(\mu)}$ for $m_{\chi} \gtrsim 2 \mathrm{MeV}$, see also Fig. 2. In addition, to illustrate the increased sensitivity of NA64 $\mu$, in Fig. 3 we plot the total cross sections (10) for muon and electron beam setup. One can see from Fig. 3 and Eq. (20), for the small mass range $m_{\chi}<m_{\mu}$ the muon cross 
section decreases slowly as $m_{\chi}$ approaches to $m_{\mu}$ in contrast to the electron beam case. On the other hand, for $m_{\chi}>m_{\mu}$ both the electron and the muon cross sections scale as $1 / m_{\chi}^{2}$ up to the logarithmic terms in (19). Given that, one can gain the increased sensitivity (18) for the muon beam setup in the large mass range $m_{\mu} \gtrsim m_{\mu}$. Namely, at $m_{\mu} \simeq 200 \mathrm{MeV}$ one has $\sigma_{\chi \chi}^{(\mu)}\left[Q_{\chi}^{(\mu)}=1\right] / \sigma_{\chi \chi}^{(e)}\left[Q_{\chi}^{(e)}=1\right] \approx 1 / 5$, and, therefore we have $Q_{\chi}^{(e)} / Q_{\chi}^{(\mu)} \approx 9$ for $L_{\text {eff }}^{(\mu)} / L_{\text {eff }}^{(e)} \approx 40$ and $N_{\text {MOT }} /$ $N_{\text {EOT }} \approx 10$, as outlined above.

The NA64e has an experience which indicates that the proposed search for new physics in missing energy events with $3 \times 10^{11}$ EOT is essentially background free due to the excellent detector capability for the identification of the initial and final state in the high-energy electron interactions in the dump Ref. [48]. The main potential sources of background are currently identified, and we do not expect a significant increase in background level after detector upgrade in the 2021 run.

The design of NA64 $\mu$ reported in Ref. [49] is also based on the experience of NA64e. A preliminary study of the hadron contaminations and detector Hermiticity with the M2 muon beam shows that the total background level is conservatively expected to be at the level $<10^{-12}$. Further improvements of this level are foreseen, which, however, will require modification of the beam line (e.g., adding of additional absorbers).

Before concluding, two remarks are in order. First, the NA64e experiment employed the optimized $100 \mathrm{GeV}$ electron beam from the $\mathrm{H} 4$ beam line at the SPS. The beam has a maximal intensity $10^{7} e^{-}$per SPS spill of $4.8 \mathrm{~s}$ produced by the primary $400 \mathrm{GeV}$ proton beam, and about 4000 good spills per day. Thus, $\sim 120$ days are required to accumulate $5 \times 10^{12}$ EOT at the $\mathrm{H} 4$ electron beam line.
Second, the intensity of $100 \mathrm{GeV}$ muons at the M2 beam line is a factor 10 higher. Thus, about the same running time of 120 days is required to accumulate $5 \times 10^{13}$ MOT. Here, the challenge might be to keep the overall efficiency still high at the intensity $\sim 10^{8}$ muons/spill, which however seems possible [50].

\section{CONCLUSIONS}

In this paper we considered the prospects of the millicharged particle discovery at the NA64 experiment at CERN in the $\mathrm{MeV}-\mathrm{GeV}$ mass range of $\chi$. These weakly coupled to photon particles can be constrained by high energy collider experiments. We studied both cases of electron and muon beams at NA64 (NA64e and NA64 $\mu$ ). We also calculate the relevant millicharge production cross sections in the equivalent photon approach. We analyze in detail the corresponding parameter space in the range of small, $m_{\chi} \ll m_{\mu}$, and large masses, $m_{\chi} \gtrsim m_{\mu}$. We found that NA64 $\mu$ can constrain the wider range of the millicharge parameter space for $m_{\chi} \gtrsim m_{\mu}$ due to the increased accumulated statistic of muons and their small attenuation rate in the target. However, both NA64e and NA64 $\mu$ will be able to compete with current and forthcoming experimental facilities. Nevertheless, a more careful Monte Carlo simulation of $\chi$ production at NA64 is required to take into account the realistic response of the NA64 detector. We leave this task for future analysis.

\section{ACKNOWLEDGMENTS}

We would like to thank P. Schuster and G. Lanfranchi for fruitful discussions.
[1] A. Yu. Ignatiev, V. A. Kuzmin, and M. E. Shaposhnikov, Phys. Lett. 84B, 315 (1979).

[2] P. A. M. Dirac, Proc. R. Soc. A 133, 60 (1931).

[3] B. Holdom, Phys. Lett. 166B, 196 (1986).

[4] L. B. Okun, Zh. Eksp. Teor. Fiz. 83, 892 (1982) [Sov. Phys. JETP 56, 502 (1982)].

[5] M. I. Dobroliubov and A. Yu. Ignatiev, Phys. Rev. Lett. 65, 679 (1990).

[6] E. Golowich and R. W. Robinett, Phys. Rev. D 35, 391 (1987).

[7] A. A. Prinz et al., Phys. Rev. Lett. 81, 1175 (1998).

[8] A. Badertscher, P. Crivelli, W. Fetscher, U. Gendotti, S. N. Gninenko, V. Postoev, A. Rubbia, V. Samoylenko, and D. Sillou, Phys. Rev. D 75, 032004 (2007).

[9] S. N. Gninenko, N. V. Krasnikov, and A. Rubbia, Phys. Rev. D 75, 075014 (2007).

[10] L. Singh et al., Phys. Rev. D 99, 032009 (2019).
[11] S. Davidson, S. Hannestad, and G. Raffelt, J. High Energy Phys. 05 (2000) 003.

[12] E. Izaguirre and I. Yavin, Phys. Rev. D 92, 035014 (2015).

[13] Z. Liu and Y. Zhang, Phys. Rev. D 99, 015004 (2019).

[14] H. Goldberg and L. J. Hall, Phys. Lett. B 174, 151 (1986).

[15] S. Davidson, B. Campbell, and D. C. Bailey, Phys. Rev. D 43, 2314 (1991).

[16] S. Davidson and M. Peskin, Phys. Rev. D 49, 2114 (1994).

[17] S. L. Dubovsky, D. S. Gorbunov, and G. I. Rubtsov, JETP Lett. 79, 1 (2004).

[18] A. D. Dolgov, S. L. Dubovsky, G. I. Rubtsov, and I. I. Tkachev, Phys. Rev. D 88, 117701 (2013).

[19] Z. Berezhiani, A. D. Dolgov, and I. I. Tkachev, Eur. Phys. J. C 73, 2620 (2013).

[20] Z. Berezhiani, A. D. Dolgov, and I. I. Tkachev, J. Cosmol. Astropart. Phys. 02 (2013) 010. 
[21] G. Magill, R. Plestid, M. Pospelov, and Y. D. Tsai, Phys. Rev. Lett. 122, 071801 (2019).

[22] S. N. Gninenko, Phys. Rev. D 89, 075008 (2014).

[23] S. N. Gninenko, N. V. Krasnikov, M. M. Kirsanov, and D. V. Kirpichnikov, Phys. Rev. D 94, 095025 (2016).

[24] A. Berlin, N. Blinov, G. Krnjaic, P. Schuster, and N. Toro, Phys. Rev. D 99, 075001 (2019).

[25] T. Åkesson et al. (LDMX Collaboration), arXiv:1808.05219.

[26] D. Banerjee et al. (NA64 Collaboration), Phys. Rev. Lett. 118, 011802 (2017).

[27] D. Banerjee et al. (NA64 Collaboration), Phys. Rev. D 97, 072002 (2018).

[28] S. N. Gninenko, D. V. Kirpichnikov, M. M. Kirsanov, and N. V. Krasnikov, Phys. Lett. B 782, 406 (2018).

[29] D. Banerjee et al. (NA64 ${ }_{\mu}$ Collaboration), Reports No. CERN-SPSC-2018-024 and No. SPSC-P-348-ADD3, 2018.

[30] S. N. Gninenko, N. V. Krasnikov, and V. A. Matveev, Phys. Rev. D 91, 095015 (2015).

[31] S. N. Gninenko and N. V. Krasnikov, Phys. Lett. B 783, 24 (2018).

[32] C. Y. Chen, J. Kozaczuk, and Y. M. Zhong, J. High Energy Phys. 10 (2018) 154.

[33] Y. Kahn, G. Krnjaic, N. Tran, and A. Whitbeck, J. High Energy Phys. 09 (2018) 153.

[34] C. Y. Chen, M. Pospelov, and Y. M. Zhong, Phys. Rev. D 95, 115005 (2017).

[35] G. W. Bennett et al. (Muon $g-2$ Collaboration), Phys. Rev. Lett. 92, 161802 (2004).
[36] S. Chatrchyan et al. (CMS Collaboration), Phys. Rev. D 87, 092008 (2013).

[37] M. Anelli et al. (SHiP Collaboration), arXiv:1504.04956.

[38] A. A. Aguilar-Arevalo et al. (MiniBooNE Collaboration), Phys. Rev. Lett. 121, 221801 (2018).

[39] R. Acciarri et al. (DUNE Collaboration), arXiv:1512.06148.

[40] C. Athanassopoulos et al. (LSND Collaboration), Nucl. Instrum. Methods Phys. Res., Sect. A 388, 149 (1997).

[41] T. Beranek, H. Merkel, and M. Vanderhaeghen, Phys. Rev. D 88, 015032 (2013).

[42] Y.S. Liu and G. A. Miller, Phys. Rev. D 96, 016004 (2017).

[43] J. D. Bjorken, R. Essig, P. Schuster, and N. Toro, Phys. Rev. D 80, 075018 (2009).

[44] S. Davidson, S. Hannestad, and G. Raffelt, J. High Energy Phys. 05 (2000) 003.

[45] A. Haas, C. S. Hill, E. Izaguirre, and I. Yavin, Phys. Lett. B 746, 117 (2015).

[46] A. Berlin, D. Hooper, G. Krnjaic, and S. D. McDermott, Phys. Rev. Lett. 121, 011102 (2018).

[47] R. Barkana, N. J. Outmezguine, D. Redigolo, and T. Volansky, Phys. Rev. D 98, 103005 (2018).

[48] D. Banerjee et al., arXiv:1906.00176.

[49] D. Banerjee et al., Reports No. CERN-SPSC-2019-002 and No. SPSC-P-359, http://cds.cern.ch/record/2653581.

[50] A. Magnon et al., Reports No. CERN-SPSC-2010-014 and No. SPSC-P-340, https://cds.cern.ch/record/1265628. 